\title{
Differential gene expression in porcine SK6 cells infected with wild-type and SAP domain-mutant foot-and-mouth disease virus
}

\author{
Zixin $\mathrm{Ni}^{1,2 \#}$, Fan Yang ${ }^{1 \#}$, Weijun $\mathrm{Cao}^{1}$, Xiangle Zhang ${ }^{1}$, Ye Jin ${ }^{1}$, Ruoqing Mao ${ }^{1}$, Xiaoli Du ${ }^{1}$, Weiwei $\mathrm{Li}^{1}$, \\ Jianhong Guo ${ }^{1}$, Xiangtao Liu ${ }^{1}$, Zixiang Zhu ${ }^{1 \bowtie}$, Haixue Zheng ${ }^{1 \bowtie}$ \\ 1. State Key Laboratory of Veterinary Etiological Biology, National Foot and Mouth Diseases Reference \\ Laboratory, Key Laboratory of Animal Virology of Ministry of Agriculture, Lanzhou Veterinary Research \\ Institute, Chinese Academy of Agricultural Sciences, Lanzhou 730046, China \\ 2. College of Veterinary Medicine, China Agricultural University, Beijing 100083, China
}

Foot-and-mouth disease virus (FMDV) is the causative agent of a highly contagious disease in livestock. The viral proteinase $L^{\text {pro }}$ of FMDV is involved in pathogenicity, and mutation of the $L^{\text {pro }}$ SAP domain reduces FMDV pathogenicity in pigs. To determine the gene expression profiles associated with decreased pathogenicity in porcine cells, we performed transcriptome analysis using next-generation sequencing technology and compared differentially expressed genes in SK6 cells infected with FMDV containing $L^{\text {pro }}$ with either a wild-type or mutated version of the SAP domain. This analysis yielded 1,853 genes that exhibited a $\geq 2$-fold change in expression and was validated by real-time quantitative PCR detection of several differentially expressed genes. Many of the differentially expressed genes correlated with antiviral responses corresponded to genes associated with transcription factors, immune regulation, cytokine production, inflammatory response, and apoptosis. Alterations in gene expression profiles may be responsible for the variations in pathogenicity observed between the two FMDV variants. Our results provided genes of interest for the further study of antiviral pathways and pathogenic mechanisms related to FMDV $L^{\text {pro }}$.

\section{KEYWORDS Foot-and-mouth disease virus (FMDV); leader protein; SAP region; transcriptome analysis}

\section{INTRODUCTION}

Foot-and-mouth disease virus (FMDV) is a positivestranded RNA virus capable of infecting a variety of domestic and wild biungulate species (Pega et al., 2013). The highly contagious foot-and-mouth disease (FMD) is caused by FMDV and is perhaps the most important lim-

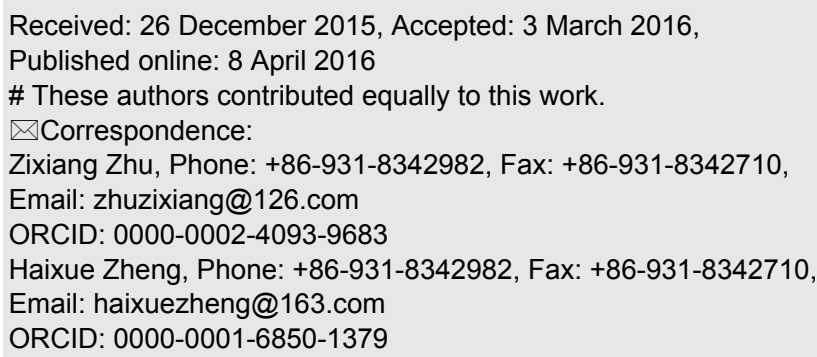

iting factor in the trade of animals and animal products (Barasa et al., 2008; Perry et al., 2007; Rufael et al., 2008), with outbreaks usually resulting in large economic losses for the local livestock industry. FMDV belongs to the Aphthovirus genus of the Picornaviridae family, and its genome is a single-stranded, positive-sense RNA that encodes a polyprotein. The polyprotein is post-translationally cleaved by three viral proteinases, leader $\left(\mathrm{L}^{\mathrm{pro}}\right)$, $2 \mathrm{~A}$, and $3 \mathrm{C}^{\text {pro }}$, into precursors and mature viral structural (VP4, VP2, VP3, and VP1) and nonstructural proteins $\left(\mathrm{L}^{\text {pro }}, 2 \mathrm{~A}, 2 \mathrm{~B}, 2 \mathrm{C}, 3 \mathrm{~A}, 3 \mathrm{~B}, 3 \mathrm{C}^{\mathrm{pro}}\right.$, and 3D) (Racaniello, 2007).

Translation of the FMDV polyprotein begins at two different AUG start codons separated by 84 nucleotides, resulting in two alternative forms of $\mathrm{L}^{\text {pro }}$ designated as $\mathrm{Lab}^{\text {pro }}$ and $\mathrm{Lb}^{\text {pro }}$ (Clarke et al., 1985; Piccone et al., 
1995). During its evolution, FMDV has consistently counteracted against host immune systems to facilitate its survival and replication; several mechanisms have evolved to antagonize host immune responses, with $\mathrm{L}^{\text {pro }}$ reported to play significant pathogenic roles (Grubman et al., 2008). $\mathrm{L}^{\text {pro }}$ is a well-characterized, papain-like proteinase (Medina et al., 1993; Piccone et al., 1995) that can self-cleave from the nascent polyprotein. Host translation-initiation factor eIF-4G can also be cleaved by $\mathrm{L}^{\text {pro }}$, greatly reducing host cap-dependent mRNA translation without affecting viral cap-independent protein synthesis, which is a characteristic of most picornavirus infections (de Los Santos et al., 2009; Devaney et al., 1988; Kirchweger et al., 1994; Zhu et al., 2010). Additionally, $\mathrm{L}^{\mathrm{pro}}$ inhibits dsRNA-induced type I interferon (IFN) transcription by inhibiting the expression of IFNregulatory factor 3/7 (Wang et al., 2010).

In eukaryotic cells, the SAP domain (scaffold-attachment factors $\mathrm{A}$ and $\mathrm{B}$, apoptotic chromatin-condensation inducer in the nucleus, and protein inhibitor of activated STAT proteins) is a putative DNA-binding domain found in diverse nuclear proteins (Aravind et al., 2000). The SAP domain consists of 35 amino acids, including conserved hydrophobic and charged residues (Aravind et al., 2000), and is found in a number of chromatin-associating proteins, such as scaffold-attachment factors, DNA-repair proteins, RNA-processing complexes, and proto-oncogene proteins (Ahn et al., 2003; Aravind et al., 2000; Bohm et al., 2005; Kipp et al., 2000).

A conserved SAP domain was also identified in the $\mathrm{L}^{\text {pro }}$-coding region of FMDV (de Los Santos et al., 2009). Genetically engineered FMDV strains lacking the $\mathrm{L}^{\text {pro }}$ coding region (leaderless viruses) or possessing a mutated $\mathrm{L}^{\text {pro }}$ SAP domain exhibited attenuated viral replication in infected cattle and swine (Chinsangaram et al., 1998; Diaz-San Segundo et al., 2012; Zhu et al., 2010). SAPdomain mutants carrying I55A and L58A substitutions abolish $\mathrm{L}^{\text {pro }}$ retention in the nuclei of FMDV-infected cells and subsequently prevent FMDV-related degradation of nuclear factor-kappa B (NF- $\kappa \mathrm{B})$ (de Los Santos et al., 2007), resulting in upregulation of several cytokines, chemokines, and IFN-stimulated genes (ISGs) (de Los Santos et al., 2009). Additionally, inoculation of swine with FMDV containing a mutated SAP domain induced early protection against disease (Diaz-San Segundo et al., 2012), and transcriptome analysis of embryonic bovine kidney cells (EBKs) infected with SAP-mutated FMDV showed enhanced expression of various IFN-related genes as compared with EBK cells infected with FMDV containing a wild-type SAP domain (de Los Santos et al., 2009).

FMDV pathogenesis presents particular features depending on the host (Pega et al., 2013). In different species, the viral entry routes, primary infective and replic- ative sites, and, consequently, the associated symptoms and immune responses elicited showed clear differences (Alexandersen et al., 2003). The different host responses triggered by FMDV also correlated with viral replication, thereby affecting viral propagation in different cells. In EBK cells, an intact $\mathrm{L}^{\text {pro }}$ SAP domain was correlated with type I IFN responses (de Los et al., 2006; Zhu et al., 2010). Here, we compared the pathogenic characteristics of FMDV containing wild-type or mutant SAP domains in swine PK15 and SK6 cells and found that the SAPmutant variant exhibited decreased pathogenicity in both cell lines, with a more pronounced decrease observed in SK6 cells, relative to the wild-type SAP variant.

To analyze the different transcription profiles induced by SAP-domain status, FMDV containing either wildtype or mutant SAP variants was used to infect SK6 cells, followed by comparative transcriptome analysis using next-generation sequencing (NGS) technology to systematically observe the differences in gene expression and host response. Of 20, 421 genes detected, differentially enhanced or repressed expression was observed in 1, 670 and 183 genes, respectively, with many associated with antiviral responses involving transcription, immune response, inflammation, apoptosis, and cytokines or chemokine production. Our results indicated that the FMDV L ${ }^{\text {pro }}$ SAP domain was significantly correlated with FMDV viral pathogenicity in SK6 cells, and that the differential expression of various host genes is dependent upon infection with FMDV containing an intact $L^{\text {pro }}$ SAP domain.

\section{MATERIALS AND METHODS}

\section{Viruses and cells}

An engineered chimeric virus, rA-FMDV, which was previously constructed by Zheng et al., was used as a candidate vaccine (Zheng et al., 2013). The rA-FMDV was constructed by replacing the $P 1$ gene in the O/CHA/99 strain (GenBank accession number: AF506822) with the P1 gene from the A/HuBWH/CHA/2009 strain (GenBank accession number: JF792355). A SAP-mutant virus, rA-SAP-FMDV, was previously constructed by Zheng et al. by introducing the I55A and L58A mutations into the SAP domain of $\mathrm{Lb}^{\text {pro }}$ in rA-FMDV (unpublished data). The schematic representation of the mutation information is shown in Figure 1A. SK6 cells were purchased from the Cell Bank of Type Culture Collection of the Chinese Academy of Sciences (Shanghai, China). The cells were maintained in Dulbecco's Modified Eagle's Medium (DMEM; Invitrogen, Carlsbad, USA) and cultured in the medium supplemented with $10 \%$ fetal bovine serum (FBS) at $37{ }^{\circ} \mathrm{C}$ under $5 \% \mathrm{CO}_{2}$. 
A

L-WT: PENLTLAAIKQLEELTGLELHEGGPPALVIWNIKHLL

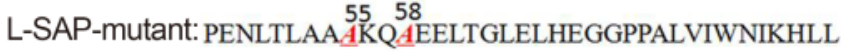

B

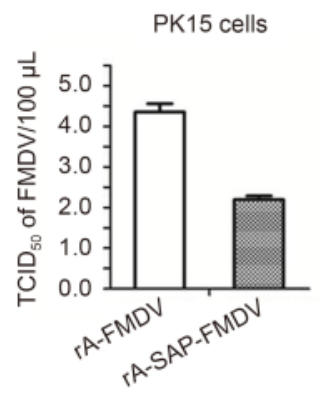

SK6 cells

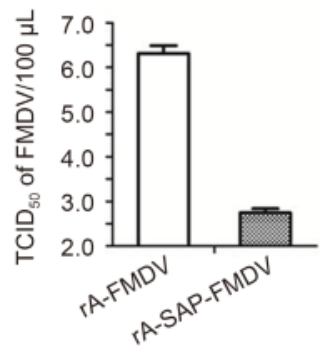

Figure 1. (A) Schematic of the I55A and L58A mutations (red, italics) in the $\mathrm{L}^{\text {pro }} \mathrm{SAP}$ domain. (B) Different pathogenicity observed between rA-FMDV and rA-SAPFMDV in SK6 and PK15 cells. SK6 and PK15 cells were infected with rA-FMDV or rA-SAP-FMDV at similar MOls, and the viral TCID $_{50}$ was detected and recorded. Results are presented as the mean \pm standard error from three independent experiments.

\section{Virus infection and $50 \%$ tissue culture infectious-dose $\left(\mathrm{TCID}_{50}\right)$ assay}

SK6 and PK15 cells were washed with phosphate-buffered saline and infected with FMDV at a multiplicity of infection (MOI) of 1 at $37^{\circ} \mathrm{C}$. After a 1-h adsorption period, the supernatant was removed, and the cells were incubated at $37^{\circ} \mathrm{C}$ with DMEM containing $0.5 \% \mathrm{FBS}$. The cells used for transcription profile analyses were harvested at 6 -h post-infection, because a minimal cytopathic effect (CPE) was observed at $\sim 6 \mathrm{~h}$, enabling a more complete identification of differentially expressed genes. The $\mathrm{TCID}_{50}$ assay was performed in 96-well plates according to standard procedures, and cells were cultured until CPEs were clearly observed (3-5 days). The TCID 50 value was calculated using the ReedMuench method (Reed et al., 1938).

\section{RNA extraction and real-time quantitative PCR (qPCR)}

Total RNA was extracted from SK6 cells using TRIzol Reagent (Invitrogen) according to manufacturer protocol. Two micrograms of total RNA was used to synthesize the first strand of cDNA using M-MLV reverse transcriptase (Invitrogen), and the synthesized cDNA were subjected to qPCR analysis performed using SYBR Premix Ex Taq (Takara, Kyoto, Japan) according to manufacturer protocol. The housekeeping gene glyceraldehyde-3-phosphate dehydrogenase was used as an internal control. Primer sequences used in this study are listed

in Supplementary Table S1. The results were obtained from three independent experiments.

\section{RNA library construction and Solexa/lllumina sequencing}

A sample-pooling strategy was performed in this study. The rA-FMDV- and rA-SAP-FMDV-infected samples were used as mixture samples, with each sample prepared by mixing four different dishes of virus-infected cells. After total-RNA extraction and DNase I treatment, magnetic beads conjugated with oligo (dT) were used to isolate mRNA. The mRNA was divided into short fragments, and cDNA was synthesized using the mRNA fragments as templates. The synthesized cDNA was purified and resolved with elution buffer $[10 \mathrm{mmol} / \mathrm{L}$ Tris- $\mathrm{Cl}$ (pH 8.5)] for end repair and single-nucleotide (adenine) addition. Subsequently, the treated fragments were connected using adapters and subjected to agarose gel electrophoresis, and suitable fragments were selected as templates for PCR amplification. The quality of the obtained library was verified using an Agilent 2100 Bioanalyzer (Agilent, Santa Clara, CA, USA) and an ABI StepOnePlus Real-Time PCR System (Applied Biosystems, Foster City, CA, USA) for quantification and qualification. The library was sequenced using an Illumina HiSeqTM 2000 (Illumina, San Diego, CA, USA), and the raw data was deposited as a National Center for Biotechnology Information (NCBI) BioProject (accession reference: PRJNA269140).

\section{Gene analysis}

To identify the host genes associated with FMDV pathogenicity in SK6 cells, the original data were filtered and screened for differentially expressed genes. The NCBInr database was selected as the analytical database, and genetic data containing adaptors and low-quality reads were excluded. The short oligonucleotide analysis package (SOAPaligner/SOAP2) (Li et al., 2008) was used to quickly and accurately align the reads generated by the Illumina/Solexa Genome Analyzer (Illumina); and the reads per kilobase transcriptome per million mapped reads [RPKM; $\mathrm{RPKM}=10^{6} \mathrm{C} /\left(\mathrm{N} \times \mathrm{L} / 10^{3}\right)$, where $\mathrm{C}$ represents the number of reads uniquely aligned to the gene of interest, $\mathrm{N}$ is the total number of reads that are uniquely aligned to all genes, and $\mathrm{L}$ is the number of bases in the gene of interest (Mortazavi et al., 2008)] was calculated. The RPKM method is able to eliminate the influence of different gene lengths and sequencing discrepancies on the calculation of gene expression. Therefore, the calculated gene expression can be used for comparing differences in gene expression among samples. A method to calculate the significance of digital gene-expression profiles was used for analysis of differentially expressed genes (Audic et al., 1997). We used a false discovery rate $(\mathrm{FDR}) \leq 0.001$ (mascot FDR calculation: http://www. 
matrixscience.com/help/decoy help.html) and an absolute value of the $\log _{2}$ Ratio $\geq 1$ as the thresholds to judge the significance of differences in gene expression. The screened differentially expressed genes were further analyzed by Gene Ontology (GO) and pathway-enrichment analysis. The GO database (http://www.geneontology.org/) and GO TermFinder software (http://smd.stanford.edu/help/ GO-TermFinder/GO_TermFinder_help.shtml/) were used to perform GO analysis, and the Kyoto Encyclopedia of Genes and Genomes database (Kanehisa et al., 2008) was used for pathway-enrichment analysis.

\section{RESULTS}

\section{Viral replication in porcine cells differs between FMDV containing wild-type or mutant SAP domain}

The mutated region in the SAP domain of rA-SAP-FMDV was confirmed by sequencing analysis. A low MOI leads to infection of a percentage of cells, resulting in these infected cells signaling adjacent, uninfected cells via cytokines in order to activate antiviral genes, including secreted innate immune proteins. To study the signal transduction pathways and proteins involved, infections in this study were performed at 1 MOI. To compare the replication status of rA-FMDV and rA-SAP-FMDV in porcine PK15 and SK6 cells, the cells were infected with equal concentrations of rA-FMDV or rA-SAP-FMDV. The samples were collected 12-h post-infection, and the titers determined by $\mathrm{TCID}_{50}$ assay. The results showed that $\mathrm{rA}-\mathrm{FMDV}$ replicated more quickly relative to $\mathrm{rA}$ SAP-FMDV in both PK15 and SK6 cells (Figure 1B), indicating that the SAP mutation decreased FMDV replication in porcine PK15 and SK6 cells, with a larger decrease observed in SK6 cells.

\section{Differentially expressed genes between SK6 cells infected with FMDV containing wild-type or mutant SAP domain}

To explore the differentially expressed genes involved in the altered pathogenicity observed in SK6 cells infected with FMDV containing the SAP mutation, rA-FMDV-infected and rA-SAP-FMDV-infected SK6 cells were collected at 6 -h post-infection, and transcriptome analysis was performed. After a stringent quality check and filtering of the data (FDR $\leq 0.001$ and fold-change $\geq 2), 20$, 421 genes were detected, with 1, 853 differentially expressed genes identified. A total of 1, 670 and 183 genes were differentially upregulated and downregulated, respectively, between rA-SAP-FMDV- and rA-FMDV-infected SK6 cells (Supplementary Figure S1, S2). The expression of 117 transcription factor-related genes involved in 12 biological processes, 114 immune regulation-related genes participating in 40 immune-regulatory processes, 69 cytokine-related genes, including 20 involved in cytokine-production and -secretion processes, 12 inflammatory response-related genes, and 19 apoptosis-related genes were significantly altered (Table 1, Supplementary Table S2-S5). The distinctively different expression profiles of these genes may explain the decreased pathogenicity observed following infection with rA-SAP-FMDV. An analysis of the available literature indicated that the majority of the differentially expressed genes correlating with antiviral responses included (Table 2): 1) genes involved in transcriptional regulation (EIF4A2, EIF5B, EIF3J, NFKBIA, and NFKBIZ); 2) genes involved in the regulation of immune response (IFIT1, ITCH, IL7R, JAK2, LTB, TNFSF10, IL7, BLM, IFIT1, IL18,IL6, and FOS); 3) cytokine-related genes (IL1, IL6, IL20, TNF, CCL2, CCL20, CXCL10, CXCL2, $C C L 3 L 1, C C L 4, C C L 5$, and $C X C L 11)$; 4) genes involved in the regulation of inflammation and chemokine production (TNF, CCL5, IL1A, IL6, IL6ST, CCL2, and $I T C H)$; and 5) genes involved in apoptosis (BLM, CASP3, BRCA2, PMAIP1, CD38, MAP3K5, CUL5, TN$F S F 10$, and XIAP).

\section{Validation of differentially expressed genes by qPCR}

To further confirm and validate the transcriptome analysis results, we performed qPCR analysis to determine the reproducibility of the differential gene expression. A se-

Table 1. Summary of differential expressed genes

\begin{tabular}{llll}
\multirow{2}{*}{ Function } & \multirow{2}{*}{ Total gene number } & \multicolumn{2}{l}{ Up- or down-regulated gene number } \\
\cline { 3 - 4 } & & Up-regulated & Down-regulated \\
\hline transcription factor-related genes & 117 & 109 & 8 \\
immune regulation-related genes & 114 & 104 & 10 \\
cytokine-related genes & 69 & 62 & 7 \\
inflammatory response-related genes & 12 & 11 & 1 \\
apoptosis-related genes & 19 & 18 & 1 \\
\hline
\end{tabular}


Table 2. List of genes that displayed significant differential expression at WT and SAP mutant FMDV-infected SK6 cells

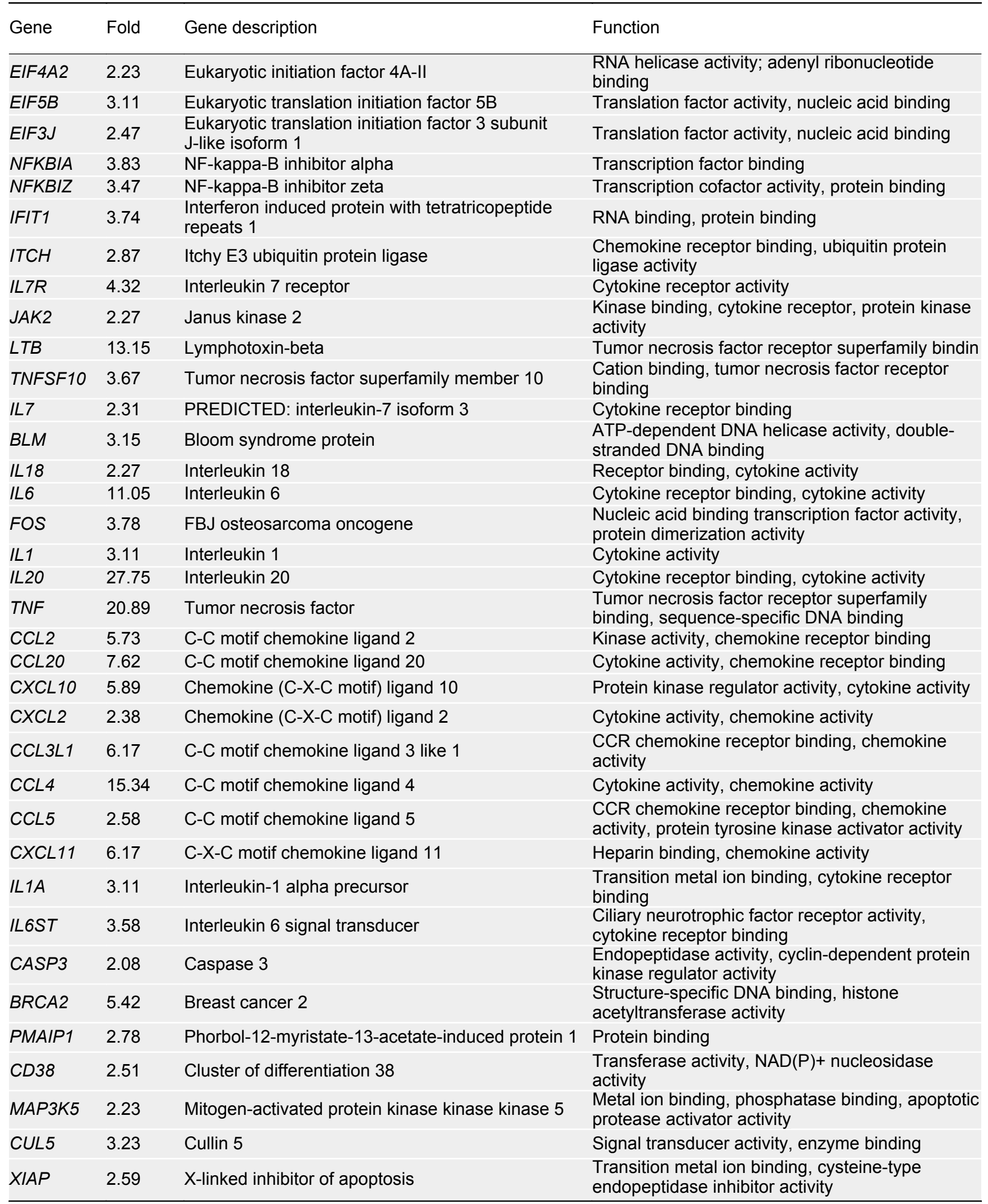

Note: A minimum of twofold change $(P<0.0001, Q<0.0001)$ was used as the standards for selecting genes of interest. 
lected group of genes for which we had an established method available in our laboratory (with established primers and melting/annealing temperatures previously) were chosen for analysis. Six upregulated genes $(C C L 4$, $C C L 2, I L 6, I L 7, I L 18$, and $E G R 1)$ and four downregulated genes (SRPX2, CREB5, RASAL1, and RIN2) were analyzed, and qPCR results confirmed the differential expression identified between rA-SAP-FMDV- and rAFMDV-infected cells. As shown in Figure 2, the qPCR results corresponded with transcriptome analysis results and, while some fold-change differences were observed between results from each method, similarities in the overall expression profiles were revealed.

\section{Functional characterization of differentially expressed genes and pathways affected by infection with FMDV containing the SAP mutation}

Infection with FMDV containing the SAP-domain mutation altered gene expression in SK6 cells, thereby affecting various biological processes and signal transduction pathways, and resulting in blocked viral replication and decreased pathogenicity. To systematically analyze the functional characterization of the differentially expressed genes and pathways associated with the FMDV $\mathrm{L}^{\text {pro }}$ SAP domain, GO analysis and pathway annotation were conducted. The results indicated that the differentially expressed genes were involved in metabolism, cell cycle processes, and cellular component organization or biogenesis processes (Supplementary Figure S3), and functional analysis revealed that many of these genes were involved in nucleotide binding and functions asso-

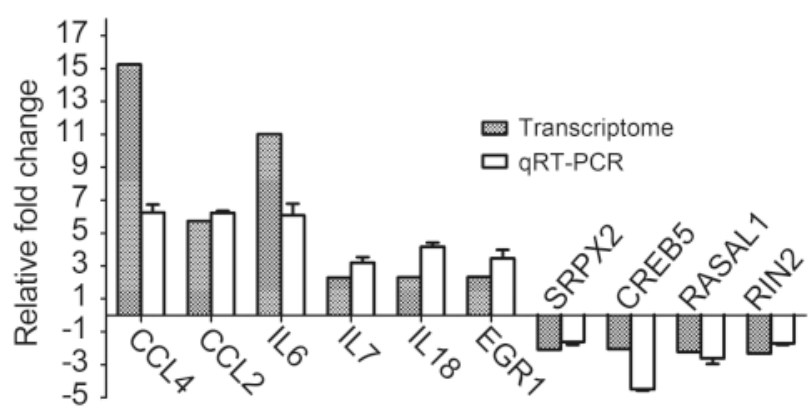

Figure 2. Validation of differentially expressed genes identified by transcriptome analysis through qPCR detection. Six upregulated and four downregulated genes were detected in an independent infection experiment undertaken in order to validate transcriptome analysis results. The expression profiles of the 10 selected genes were consistent between the transcriptome-analysis and qPCR-detection results. Results are presented as the mean \pm standard error from three independent experiments. ciated with nucleic acids (Supplementary Figure S4). Cellular component annotation results are shown in Supplementary Figure S5.

Pathway analysis indicated that 35 pathways were altered in rA-SAP-FMDV-infected cells as compared with $\mathrm{rA-FMDV}$-infected cells, including regulation of actin cytoskeleton formation, endocytosis, phagosome formation, chemokine-signaling pathways, the cell cycle, the retinoic acid-inducible gene 1-like receptor signaling pathway, the NF- $\mathrm{BB}$ signaling pathway, and the nucleotide-binding oligomerization domain-like receptor signaling pathway (Supplementary Figure S6). The potential host targets for $\mathrm{L}^{\text {pro }}$ and the protein-protein interaction pathways involved are shown in Figure 3. These findings suggested that expression of these genes might potentially result in decreased rA-SAP-FMDV replication.

\section{DISCUSSION}

FMDV has the ability to manipulate various host-cell signal transduction pathways by subverting gene expression. FMDV L ${ }^{\text {pro }}$ is a host-cell antagonist that interferes with host gene expression, promotes viral propagation, and subverts host immune systems by targeting eIF-4G, IRF3, IRF7, and NF-кB (de Los Santos et al., 2009; Kirchweger et al., 1994; Wang et al., 2010). The FMDV $\mathrm{L}^{\text {pro }} \mathrm{SAP}$ domain is a putative DNA-binding domain found in diverse eukaryotic nuclear proteins (Aravind et al., 2000; de Los Santos et al., 2009) and inhibits host innate immune response (de Los Santos et al., 2009). The mutation of two amino acids (I55A and L58A) in the $\mathrm{Lb}^{\text {pro }}$ SAP domain alters $\mathrm{L}^{\text {pro }}$ subcellular localization and function (de Los Santos et al., 2009; Diaz-San Segundo et al., 2012).

In a previous study, we constructed a chimeric virus, rA-FMDV, as a candidate vaccine (Zheng et al., 2013). To decrease rA-FMDV pathogenicity and develop a potential live attenuated vaccine, we constructed rA-SAPFMDV containing a mutated SAP domain (unpublished data). Here, we compared the different pathogenic characteristics of rA-FMDV and rA-SAP-FMDV in PK15 and SK6 cells, and showed that rA-SAP-FMDV was less pathogenic relative to rA-FMDV in both cell lines, suggesting that rA-SAP-FMDV may have potential as a vaccine strain based on its failure to disrupt cellular responses that inhibit viral replication.

Decreased rA-SAP-FMDV replication was more evident in SK6 cells as compared with PK15 cells. To analyze the differentially expressed genes correlated with the altered viral replication observed in SK6 cells, transcriptome analysis was performed using NGS technology. The results indicated differential expression of 


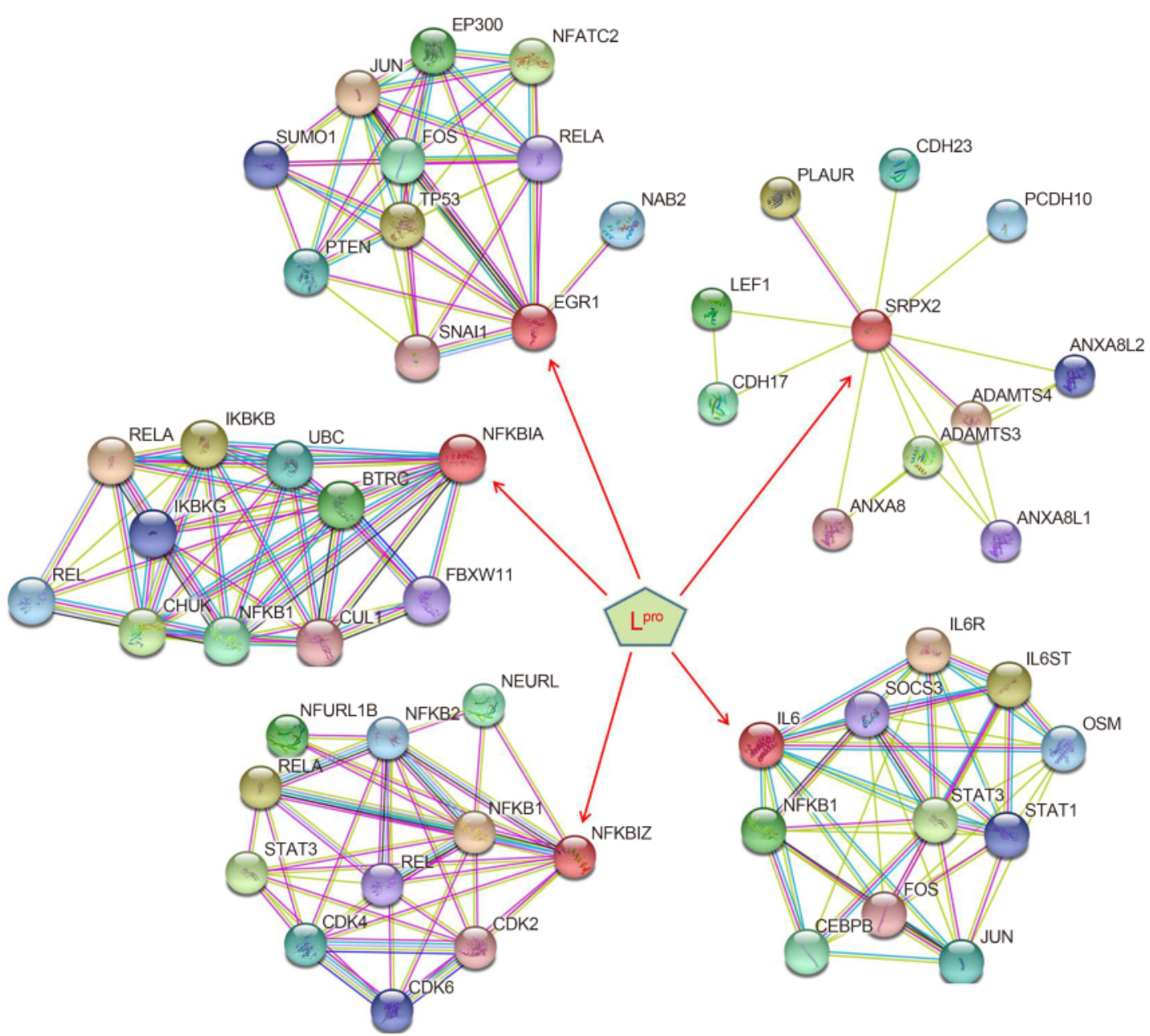

Figure 3. The potential host targets of $\mathrm{L}^{\text {pro }}$ and the involved protein-protein interaction pathways.

1, 853 genes between infected and non-infected SK6 cells, with these findings subsequently confirmed by qP$\mathrm{CR}$ analysis. These findings suggested that mutation of the FMDV L ${ }^{\text {pro }}$ SAP domain might adversely affect the ability of FMDV to inhibit host gene expression during infection, resulting in reduced viral pathogenicity.

Among the differentially expressed genes upregulated in rA-SAP-FMDV-infected cells, EIF4A2, EIF5B, and $E I F 3 J$ are involved in the initiation of host translation by aiding in the recruitment of protein and mRNA components to ribosomes (Cheyssac et al., 2006; ElAntak et al., 2007; Kyono et al., 2002; Meijer et al., 2013; Unbehaun et al., 2007). Swine infected with FMDV containing a mutated SAP domain developed a strong neutralizing-antibody response as early as 2-days post-inoculation as compared with those infected with wild-type FMDV (Diaz-San Segundo et al., 2012). The upregulation of these translation factors possibly resulted in the enhancement of neutralizing-antibody production. Furthermore, our analysis revealed upregulation of other genes, including those involved in metabolic and cellular-response processes (Supplementary Figure S3).

FMDV infection can induce degradation of NF- $\kappa \mathrm{B}$ (de Los Santos et al., 2007); however, NF-אB activity was significantly enhanced in cells infected with FMDV containing a mutated SAP domain (Zhu et al., 2010). NFK$B I A$ and $N F K B I Z$ are involved cytokine production through NF- $\kappa B$ regulation (Ninomiya-Tsuji et al., 1999; Yamazaki et al., 2001). In this study, we found that the expression of NFKBIA and NFKBIZ was upregulated in rA-SAP-FMDV-infected cells, which may have altered the subsequent expression of NF- $\mathrm{\kappa B}$-induced cytokines to ensure a robust immune response (Figure 3). CCL4 and $I L 7$, both involved in cellular immune and inflammatory responses, were also upregulated in these cells. Additionally, the upregulation of many other genes involved in immune response, inflammation, chemokine production, and apoptosis was also observed.

Upregulation of EGRI and IL6 expression was also observed in rA-SAP-FMDV-infected cells. EGR1 mediates cell proliferation, differentiation, inflammation, and apoptosis (Han et al., 2015), and may interact with p53 
or FOS to regulate apoptosis or transcription (Figure 3). IL6 is involved in inflammation, B cell maturation, and suppression of viral replication (Dienz et al., 2012). Here, upregulation of IL 6 expression possibly enhanced acute-phase response and suppressed rA-SAP-FMDV replication. Our results suggested that $\mathrm{L}^{\mathrm{pro}}$ may attenuate EGRI and IL6 expression and adversely affect regulation of cell proliferation, transcription, differentiation, inflammation, immune response, and apoptosis to promote viral replication. Furthermore, mutation of the $\mathrm{L}^{\text {pro }} \mathrm{SAP}$ domain might impair this antagonistic effect, thereby inhibiting viral replication (Figure 3). The resulting upregulation of these genes likely reinforced the antiviral activity of the cells, directly resulting in the decreased pathogenicity observed following rA-SAP-FMDV infection. Conversely, downregulation of $S R P X 2$, which is involved in anti-apoptotic activity, was observed in rASAP-FMDV-infected SK6 cells (Figures 2 and 3). This indicated an alternative pathway for suppressing viral replication through the promotion of cell death. Intact $\mathrm{L}^{\mathrm{pro}}$ likely inhibits the initiation of apoptosis, with our results suggesting that mutation of the $\mathrm{L}^{\text {pro }}$ SAP domain impaired this inhibitory effect.

A previous study found that in EBK cells infected with a FMDV containing the SAP-domain mutation, NF$\kappa \mathrm{B}$ was the primary factor responsible for the differential transcription of many upregulated genes associated with innate immune response (Zhu et al., 2010). Here, we found that the differential gene expression observed in rA-SAP-FMDV-infected SK6 cells resulted from altered expression of genes involved in transcription and immune-related regulation. However, IFN-stimulated genes, such as $I S G 15$, ISG 20, MXI, GBP1, and $O A S 1$, which were differentially expressed in EBK cells infected with a FMDV containing the SAP-domain mutation, were not observed in this study. This is possibly due to SK6 cells being deficient in type I IFN production (Ruggli et al., 2003), although the enhancement of various type I IFN-independent genes and pathways determined in this study were implied to perform crucial antiviral effects.

In summary, we reported that a FMDV L ${ }^{\text {pro }}$ SAP-domain mutant exhibited decreased replication ability in SK6 cells as compared with wild-type FMDV. Transcriptome analysis suggested that the altered expression of genes involved in transcription, immune response, cytokine and chemokine production, inflammation, and apoptosis were the primary reasons for the observed decrease in pathogenicity. Our results provided insight into the pathogenic mechanisms associated with the FMDV $\mathrm{L}^{\text {pro }} \mathrm{SAP}$-domain and suggested that mutation of region provides a strategy for the development new FMDV-related vaccines having impaired host-antagonistic ability.

\section{ACKNOWLEDGMENTS}

We thank Dr. Jinwen Liu for providing valuable technical assistance and suggestions. This work was supported by grants from the National Science and Technology Ministry (2015BAD12B04), National Natural Sciences Foundation of China (No. 31302118, 31502042 and 31402179), the Gansu Science Foundation for Distinguished Young Scholars (no. 145RJDA328), the International Atomic Energy Agency (16025/R0) and the Key technologies R\&Dprogram of Gansu Province (1302NKDA027).

\section{COMPLIANCE WITH ETHICS GUIDELINES}

The authors declare that they have no conflict of interest. This article does not contain any studies with human oranimal subjects performed by any of the authors.

\section{AUTHOR CONTRIBUTIONS}

ZXN, FY, ZXZ and HXZ designed the research, ZXN, FY, ZXZ, XD, WWL and WJC performed the experiments. XLZ, YJ, JHG and XTL provided experiment support. ZXN, ZXZ and HXZ wrote the manuscript. All authors have read and approved the final manuscript for submission.

Supplementary figures/tables are available on the website of Virologica Sinica: www.virosin.org; link.springer. com/journal/12250.

\section{REFERENCES}

Ahn JS, Whitby MC. 2003. The role of the SAP motif in promoting Holliday junction binding and resolution by SpCCE1. J Biol Chem, 278: 29121-29129.

Alexandersen S, Zhang Z, Donaldson AI, Garland AJ. 2003. The pathogenesis and diagnosis of foot-and-mouth disease. J Comp Pathol, 129: 1-36.

Aravind L, Koonin EV. 2000. SAP-a putative DNA-binding motif involved in chromosomal organization. Trends Biochem Sci, 25: 112-114.

Audic S, Claverie JM. 1997. The significance of digital gene expression profiles. Genome Res, 7: 986-995.

Barasa M, Catley A, Machuchu D, Laqua H, Puot E, Tap KD, Ikiror D. 2008. Foot-and-mouth disease vaccination in South Sudan: benefit-cost analysis and livelihoods impact. Transbound Emerg Dis, 55: 339-351.

Bohm F, Kappes F, Scholten I, Richter N, Matsuo H, Knippers R, Waldmann T. 2005. The SAF-box domain of chromatin protein DEK. Nucleic Acids Res, 33: 1101-1110. 
Cheyssac C, Dina C, Lepretre F, Vasseur-Delannoy V, Dechaume A, Lobbens S, Balkau B, Ruiz J, Charpentier G, Pattou F, Joly E, Prentki M, Hansen T, Pedersen O, Vaxillaire M, Froguel P. 2006. EIF4A2 is a positional candidate gene at the $3 q 27$ locus linked to type 2 diabetes in French families. Diabetes, 55: 1171-1176.

Chinsangaram J, Mason PW, Grubman MJ. 1998. Protection of swine by live and inactivated vaccines prepared from a leader proteinase-deficient serotype A12 foot-and-mouth disease virus. Vaccine, 16: 1516-1522.

Clarke BE, Sangar DV, Burroughs JN, Newton SE, Carroll AR, Rowlands DJ. 1985. Two initiation sites for foot-and-mouth disease virus polyprotein in vivo. J Gen Virol, 66: 2615-2626.

de Los Santos T, Diaz-San Segundo F, Grubman MJ. 2007. Degradation of nuclear factor kappa B during foot-and-mouth disease virus infection. J Virol, 81: 12803-12815.

de Los Santos T, Diaz-San Segundo F, Zhu J, Koster M, Dias CC, Grubman MJ. 2009. A conserved domain in the leader proteinase of foot-and-mouth disease virus is required for proper subcellular localization and function. J Virol, 83: 1800-1810.

de Los ST, de Avila BS, Weiblen R, Grubman MJ. 2006. The leader proteinase of foot-and-mouth disease virus inhibits the induction of beta interferon mRNA and blocks the host innate immune response. J Virol, 80: 1906-1914.

Devaney MA, Vakharia VN, Lloyd RE, Ehrenfeld E, Grubman MJ. 1988. Leader protein of foot-and-mouth disease virus is required for cleavage of the p220 component of the cap-binding protein complex. J Virol, 62: 4407-4409.

Diaz-San Segundo F, Weiss M, Perez-Martin E, Dias CC, Grubman MJ, de Los Santos T. 2012. Inoculation of swine with footand-mouth disease SAP-mutant virus induces early protection against disease. J Virol, 86: 1316-1327.

Dienz O, Rud JG, Eaton SM, Lanthier PA, Burg E, Drew A, Bunn J, Suratt BT, Haynes L, Rincon M. 2012. Essential role of IL-6 in protection against $\mathrm{H} 1 \mathrm{~N} 1$ influenza virus by promoting neutrophil survival in the lung. Mucosal Immunol, 5: 258-266.

ElAntak L, Tzakos AG, Locker N, Lukavsky PJ. 2007. Structure of eIF3b RNA recognition motif and its interaction with eIF3j: structural insights into the recruitment of eIF3b to the $40 \mathrm{~S}$ ribosomal subunit. J Biol Chem, 282: 8165-8174.

Grubman MJ, Moraes MP, Diaz-San Segundo F, Pena L, de Los Santos T. 2008. Evading the host immune response: how footand-mouth disease virus has become an effective pathogen. FEMS Immunol Med Microbiol, 53: 8-17.

Han H, Xue-Franzen Y, Miao X, Nagy E, Li N, Xu D, Sjoberg J, Bjorkholm M, Claesson HE. 2015. Early growth response gene (EGR)-1 regulates leukotriene D4-induced cytokine transcription in Hodgkin lymphoma cells. Prostaglandins Other Lipid Mediat, 121: 122-130.

Kanehisa M, Araki M, Goto S, Hattori M, Hirakawa M, Itoh M, Katayama T, Kawashima S, Okuda S, Tokimatsu T, Yamanishi Y. 2008. KEGG for linking genomes to life and the environment. Nucleic Acids Res, 36: D480-D484.

Kipp M, Gohring F, Ostendorp T, van Drunen CM, van Driel R, Przybylski M, Fackelmayer FO. 2000. SAF-Box, a conserved protein domain that specifically recognizes scaffold attachment region DNA. Mol Cell Biol, 20: 7480-7489.

Kirchweger R, Ziegler E, Lamphear BJ, Waters D, Liebig HD, Sommergruber W, Sobrino F, Hohenadl C, Blaas D, Rhoads RE, Et A. 1994. Foot-and-mouth disease virus leader proteinase: purification of the $\mathrm{Lb}$ form and determination of its cleavage site on eIF-4 gamma. J Virol, 68: 5677-5684.

Kyono K, Miyashiro M, Taguchi I. 2002. Human eukaryotic initiation factor 4AII associates with hepatitis C virus NS5B protein in vitro. Biochem Biophys Res Commun, 292: 659-666.
Li R, Li Y, Kristiansen K, Wang J. 2008. SOAP: short oligonucleotide alignment program. Bioinformatics, 24: 713-714.

Medina M, Domingo E, Brangwyn JK, Belsham GJ. 1993. The two species of the foot-and-mouth disease virus leader protein, expressed individually, exhibit the same activities. Virology, 194: 355-359.

Meijer HA, Kong YW, Lu WT, Wilczynska A, Spriggs RV, Robinson SW, Godfrey JD, Willis AE, Bushell M. 2013. Translational repression and eIF4A2 activity are critical for microRNA-mediated gene regulation. Science, 340: 82-85.

Mortazavi A, Williams BA, McCue K, Schaeffer L, Wold B. 2008. Mapping and quantifying mammalian transcriptomes by RNA-Seq. Nat Methods, 5: 621-628.

Ninomiya-Tsuji J, Kishimoto K, Hiyama A, Inoue J, Cao Z, Matsumoto K. 1999. The kinase TAK1 can activate the NIK-I kappaB as well as the MAP kinase cascade in the IL-1 signalling pathway. Nature, 398: 252-256.

Pega J, Bucafusco D, Di Giacomo S, Schammas JM, Malacari D, Capozzo AV, Arzt J, Perez-Beascoechea C, Maradei E, Rodriguez LL, Borca MV, Perez-Filgueira M. 2013. Early adaptive immune responses in the respiratory tract of foot-and-mouth disease virus-infected cattle. J Virol, 87: 2489-2495.

Perry BD, Rich KM. 2007. Poverty impacts of foot-and-mouth disease and the poverty reduction implications of its control. Vet Rec, 160: 238-241.

Piccone ME, Rieder E, Mason PW, Grubman MJ. 1995. The footand-mouth disease virus leader proteinase gene is not required for viral replication. J Virol, 69: 5376-5382.

Piccone ME, Zellner M, Kumosinski TF, Mason PW, Grubman MJ. 1995. Identification of the active-site residues of the L proteinase of foot-and-mouth disease virus. J Virol, 69: 4950-4956.

Racaniello VR. 2007. Picornaviridae: The Viruses and Their Replication. In: Fields virology (5th ed). Philadelphia: Lippin-cottRaven, pp. 795-948.

Reed LJ, Muench H. 1938. A simple method of estimating fifty percent endpoints. American journal of epidemiology, 27: 493-497.

Rufael T, Catley A, Bogale A, Sahle M, Shiferaw Y. 2008. Foot and mouth disease in the Borana pastoral system, southern Ethiopia and implications for livelihoods and international trade. Trop Anim Health Prod, 40: 29-38.

Ruggli N, Tratschin JD, Schweizer M, McCullough KC, Hofmann MA, Summerfield A. 2003. Classical swine fever virus interferes with cellular antiviral defense: evidence for a novel function of N(pro). J Virol, 77: 7645-7654.

Unbehaun A, Marintchev A, Lomakin IB, Didenko T, Wagner G, Hellen CU, Pestova TV. 2007. Position of eukaryotic initiation factor eIF5B on the $80 \mathrm{~S}$ ribosome mapped by directed hydroxyl radical probing. EMBO J, 26: 3109-3123.

Wang D, Fang L, Luo R, Ye R, Fang Y, Xie L, Chen H, Xiao S. 2010. Foot-and-mouth disease virus leader proteinase inhibits dsRNA-induced type I interferon transcription by decreasing interferon regulatory factor $3 / 7$ in protein levels. Biochem Biophys Res Commun, 399: 72-78.

Yamazaki S, Muta T, Takeshige K. 2001. A novel IkappaB protein, IkappaB-zeta, induced by proinflammatory stimuli, negatively regulates nuclear factor-kappaB in the nuclei. J Biol Chem, 276: 27657-27662.

Zheng H, Guo J, Jin Y, Yang F, He J, Lv L, Zhang K, Wu Q, Liu X, Cai X. 2013. Engineering foot-and-mouth disease viruses with improved growth properties for vaccine development. PLoS One, 8: e55228.

Zhu J, Weiss M, Grubman MJ, de Los Santos T. 2010. Differential gene expression in bovine cells infected with wild type and leaderless foot-and-mouth disease virus. Virology, 404: 32-40. 